\title{
REGISTRO ELETROMIOGRAFICO DO FENÔMENO DE STRÜMPELL APLICADO AO DIAGNÓSTICO DA SINDROME PIRAMIDAL
}

\author{
JOSE L. ALONSO*
}

\begin{abstract}
RESUMO - Depois de observar há vários anos que o registro eletromiográfico (EMG) do fenômeno de Strimpell (FS) é útil para diagnosticar sindromes piramidais, a partir de marco-1987, o autor passou a pesquisar o FS sistematicamente durante a EMIG dos mussulog tibiais anteriores. Realiza esse registro com eletrodo concêntrico de agulha e calibracăo de 0,05 a $0,2 \mathrm{mV}$ e $1 s$ por divisăo. Ao paciente, em decúbito dorsal, é solicitado realizar extensão vagarosa e completa da coxa. O FS positivo caracteriza-se por EMG de contração tơnica involuntária, de amplitude crescente, do músculo t1bial anterior, simultaneamente à extensão da coxa. Com a finalidade de sistematizar a EMG dessa sincinesia e de avaliar sua utilidade no diagnóstico da síndrome piramidal, o autor revisou os 579 relatórios de EMG realizados a partir de março-1987. Foram encontrados 26 (4,49\%) relatórios de pacientes com FS positívo, que serviu para estabelecer ou confirmar que esses pacientes apresentavam sindromes piramidais. Em 20 pessoas de idađe parecida e sem sinais de liberação plramídal, a pesquisa do FS se acompanhava de silêncio eletromiográfico $(90 \%)$ ou de algumas contraçzes clónicas (10\%) do tibial anterior. O autor recomenda a inclusão da pesquisa de FS no exame EMG convencional dog tibiais anteriores, especialmente em casos de esclerose lateral amiotrófica e irente a sindromes mielorradiculares ou medulares.
\end{abstract}

\section{Electromyographic registration of the Strimpell phenomenon applied to pyramidal syndrome diatnosia.}

SUMMARY - After some years of empirical observation which pointed to the possibility of using the Strumpell phenomenon (SPh) electromyographic register (EMG) as a helpful tool in pyramidal syndrome diagnosis, the author began to study the SPh systematically, from March 1987 on, during the EMG examination of the anterior tibial muscles. The EMG was always effected with a needle concentric electrode and a calibration of 0.05 to $0.2 \mathrm{mV}$ and 1s per division. The patient, lying on the back, is asked to slowly and completely stretch his thigh, and a positive SPh displays an EMG which shows unvoluntary tonic contractions of growing amplitude on the anterior tibial muscle during its extension. In order to establish an EMG systematic schedule of this synkinesia and to be able to evaluate its relevance for pyramidal syndrome diagnosis, the author revised all 579 EMG registers he had established from March-1987 on, 26 reports (4.49\%) belonged to SPh-positive patients, and it was possible to detect or to confirm that they presented a pyramidal syndrome. 20 patients of similar age who showed no pyramidal sign presented EMG silence (90\%) or some clonic contractions (10\%) on the tibial muscle during SPh research. The author suggests, therefore, the inclusion of SPh research into the conventional EMG examination of the anterior tibial muscles, specially in cases of amyotrophic lateral sclerosis and in cases presenting myeloradicular or spinal cord syndromes.

As lesões da via piramidal, de acordo com Barraquer-Bordás 4, se acompanham de duas ordens diferentes de sintomas: deficitários (negativos ou diretos) e de liberação (positivos ou indiretos), situando entre os últimos as sincinesias. O diagnóstico

Hospital do Servidor Público Estadual de Sāo Paulo (HSPE): * Chefe do Setor de Eletromiografia. Trabalho apresentado no $X$ Congresso Brasileiro de Eletrencefalografia a Neurofisiologia Clínica (Brasilia, 11-14 outubro, 1987). 
clinico da sindrome piramidal pode tornar-se difícil, como comenta Castaigne 5 , "em consequencia da variedade de sinais a que pode dar lugar, como a paraplegia flácida por choque espinhal, a hemiplegia espástica secundária a lesão antiga capsular ou os espasmos em flexão associados a certas lesões crônicas medulares»; «por outro lado a síndrome piramidal pode depender de causas agudas (trauma, acidente vascular) ou apresentar evolução crônica»; «sua avaliação clínica é mais difícil quando a sindrome piramidal se apresenta incompleta pois, por exemplo, a motricidade voluntária pode ser normal e também os reflexos proprioceptivos serem normais ou estarem ausentes». As perturbaçōes da motricidade secundárias à síndrome piramidal podem ser registradas e objetivadas mediante a eletromiografia (EMG)1,2,7-11,13,15 (Fig. 1). Dentre essas perturbaçōes, a sincinesia de coordenação, conhecida como fenômeno do
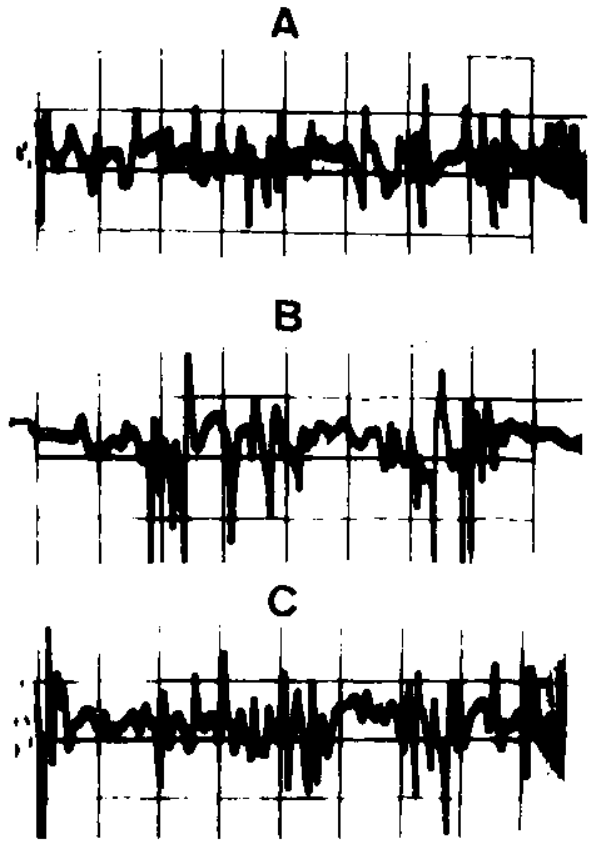

Fig. 1 - Sinais de liberacäo piramidal provocados no músculo tibial anterior. A, trasado voluntário de máximo esforço (calibracajo $0,5 \mathrm{mV}$ $e$ 20ms/div). $B$, tracado de origem reflexa provocado pela excitacão da planta do pé, coincidente a um sinal de Babinski (calibracăo $0,2 m V$ e gams/div). $C$, contração reflexa ou sincinesia provocada pela manobra de Strümpell (calibração $0,2 m V$ e 20ms/div). Alonso, 1986 (reproduçăo au:orizada pelo Editor).

tıbial ou de Strümpell (FS) 4,14 é facilmente registrada mediante a EMG e extremamente útil no diagnóstico dessa síndrome 1,2 . Barraquer-Bordás 4, baseado em critério clínico, opina que dentro dos sintomas de liberação piramidal, o FS traduz fielmente perturbações da via piramidal. Strümpell 14, em 1924, destacava o interesse diagnóstico do sinal que leva hoje seu nome: «devemos fazer menção aos movimentos associados; entende-se por tais, a transmissão patológica da inervação motora voluntária a outros territórios musculares cuja inervação não funciona. Só tem interesse diagnóstico o denominado fenômeno tibial, isto é, o aparecimento como movimento associado de pronunciada flexão do pé e proeminência do tendão do tibial anterior, durante a flexão de toda a extremidade. Este sintoma indica enfermidade orgânica das vias piramidais (hemiplegia cerebral e espinhal)». Ainda que sejam numerosos os trabalhos de neurofisiologia clínica voltados ao estudo do sistema piramidal e extrapiramidal, nota-se pouco interesse no estudo das sincinesias. Assim, por exemplo, não encontrei referências ao estudo eletrofisiológico desses movimentos anormais em manuais clássicos 8 e recentes $12,13,15$, o que contrasta com o interesse por esses sinais de liberação piramidal nos manuais de neurologia clínica 4,14. Por esses motivos, a partir de março-1987, passei a pesquisar sistematicamente o FS nos pacientes em que é necessário o estudo EMG dos músculos tibiais anteriores e nos quais a EMG e técnicas complementares não mostram alterações neurogênicas que as justifiquem.

A finalidade deste estudo é divulgar a experiência adquirida no diagnóstico EMG da síndrome piramidal mediante a pesquisa do FS e, assim, colaborar para a sistematização e interpretação desse sintoma. 


\section{MATERIAL E MTODOS}

Foram revisados 579 relatórios de EMG realizados durante o periodo de 19-marco a 47-agosto-1987, encontrando-se 26 relatórios $(4,49 \%)$ de pacientes nos quais foi constatado - FS em um ou em ambos tibiais anteriores. Este grupo, que denominarel de doentes, estava constituido de 12 hcmens e 14 mulheres, de 18 a 80 anos de idade (52 \pm 16 anos). Para servir de controle, durante o periodo de 29-junho a 5-outubro-1987, foi pesquisado o Fs nos tibiais anteriores de 20 pessoas com sintomas sensitivos subjetivos localizados em membros superiores a sem sinais clinicos piramidais. Este grupo controle era constituido de 6 homens e 14 mulheres, de 31 a 72 anos de idade (51+12 anos).

A pesquisa do FS era realizada depois do exame de EMG convencional dos tibiais anteciores. mantendo-se o paciente em decúbito dorsal e pedindo-lhe que realizasse extensão vagarosa e completa da coxa, ao mesmo tempo em que era registrado o EMG do tibial anterior do mesmo lado (Figs, 2 e 3). Esse registro era realizado com eletrodo concêntrico de agulha DANTEC 13L51, ligado a pré-amplificador Polimed conectado a osciloscópio HewlettPackard de memória e persistencia variável, modelo 180A/AR. O pré-amplificador era ajustado com limites de frequência de $10 \mathrm{~Hz}-1 \mathrm{Kz}$ e calibrado o osciloscópio com 0,05 a $0,2 \mathrm{mV}$ e 1s por divisåo. Foram anotadas as queixas, sintomas e diagnósticos clínicos que constavam nos relatórios de EMG do grupo de doentes, assim como outros sinais piramidals diferentes di) ES registrados durante esses exames.

\section{RESULTATOS}

Em 18 (90\%) pessous do grupo controle não foi registrada atividade EM: nos tibiais anteriores durante a pesquisa da prova de Strümpell. Em dois casos (10\%) foram observadas contracôes clônicas no tibial anterior durante a execuçåo dessa manobra. Resultados semelhantes foram observados no lado normal de alguns casos do grupo de doenteg (Fig. 2).

O FS estava presente no grupo de doentes e manifestava-se sob forma de FMG com aspecto de contraçōes tônicas de amplitude crescente durante o início do movimento de extensão voluntária da coxa, até alcançar amplitude máxima a partir da metade final desse movimento (Fig. 2). Simultaneamente ao EMG involuntário do tibial anterior, observava-se em alguns casos, a extensão do pé do mesmo lado (Fig. 2). Dezoito (69\%) desses pacientes

Sintomas sensitivos subjetivos Sintomas deficitários piramidaís Sintomas de liberaçåo piramídal

\begin{tabular}{|c|c|c|c|c|}
\hline Dores em MI & 7 & Hemiparesia & 4 & Sincinesias de imitaçăo \\
\hline Frio em Mi & 6 & & & Reflexos exaltados \\
\hline Parestesias & 2 & $\mathbf{E m} \mathbf{M I}$ & 3 & $\begin{array}{l}\text { Trepidaçōes } \\
\text { Marcha espástica }\end{array}$ \\
\hline Dor cervical & 2 & & & Espasticidade EMG \\
\hline Dor lombar & $\mathbf{1}$ & Em MS & 6 & Tríplice flexão \\
\hline Total & 18 & Total & 13 & Total \\
\hline
\end{tabular}

Zabela 1 - Sintomas observados em 26 pacientes que apresentavam o fenomeno de Strïmpell pasitivo, constatado a EMG: sensitivos subjetivos, em 18 (69\%); deficitarios pinamidais (moderados ou subjetivos), em 13 (50\%); de liberaçāo piramidal (observados clinicamente

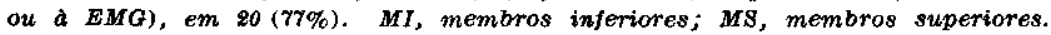

\begin{tabular}{lc}
\hline Diagnóstico & $\begin{array}{c}\text { Número de } \\
\text { casos }\end{array}$ \\
\hline \hline Esclerose múltipla & 1 \\
Esclerose lateral amiotrófica & 1 \\
Diabetes & 5 \\
Mastectomia por Ca & 1 \\
Polirradiculoneurite & 1 \\
\hline
\end{tabular}

Tabela \& - Diagnósticos clinicos, antes de reatizarem EMG, em 9 dos 26 pacientes que apresentaram o fenomeno de strïmpell a $E M G$. 

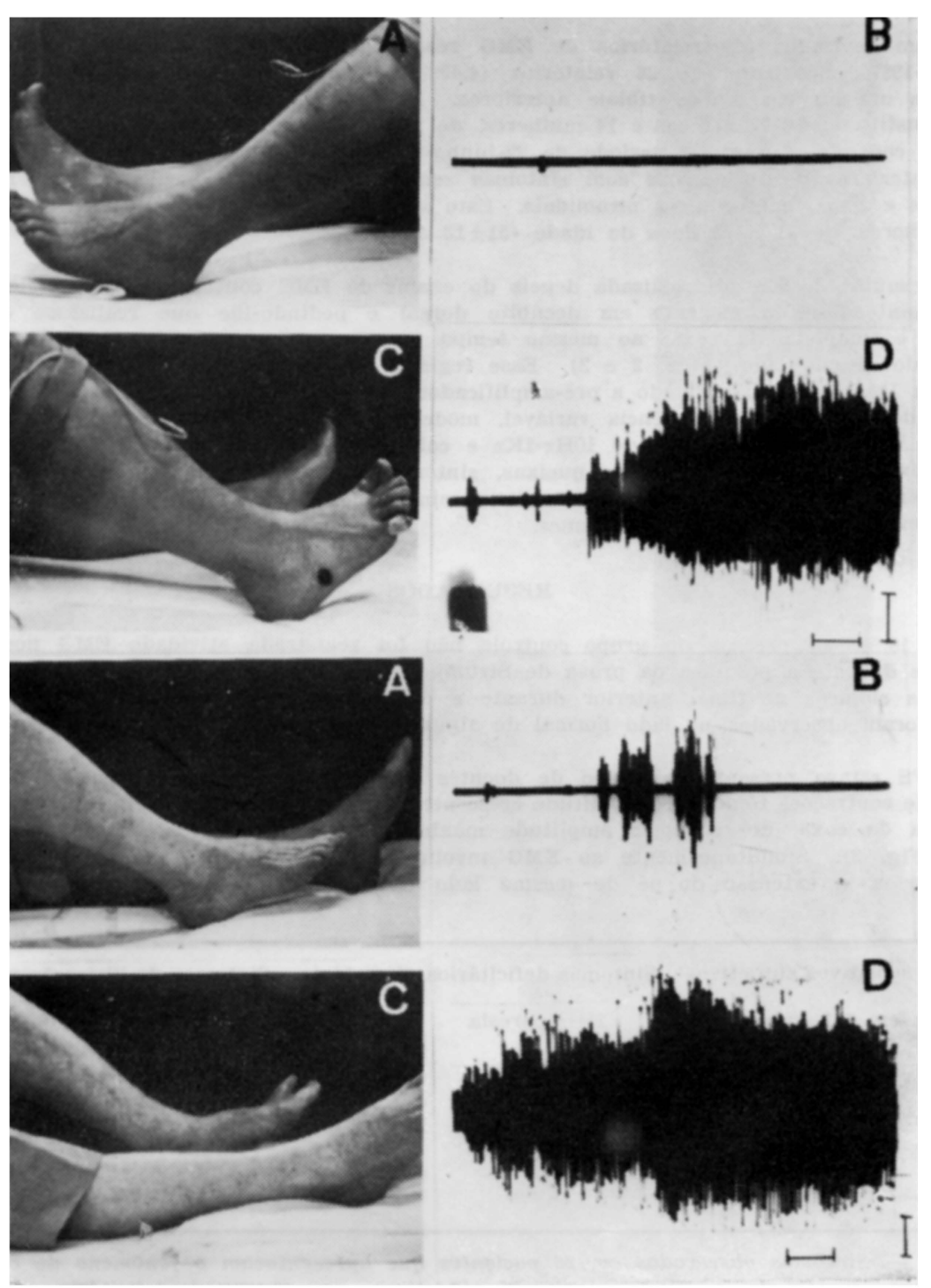

Fig. 2 - No alto: a extensão voluntäria da coxa esquerda não provoca extensão do pé (A) e nem atividade EMG no tibial anterior homolateral (B); o mesmo movimento voluntário no lado direito provoca extensão involuntária do pé (C) e, simultaneamente, registra-se atividade EMG tônica de amplitude crescente no tibial anterior homolateral (D) ou fenomeno de strümpell positivo (calibração: $0,20 \mathrm{mV}, 1 \mathrm{~s}$ ). Em baixo: a extensão voluntária da coxa direita não provoca extensão do pé (A), observiando-se simultaneamente algumast contraçóes clonicas no EMG do tibial anterior do mesmo lado $(B)$; o mesmo movimento no lado esquerdo provoca extensão involuntaria do pé (C), acompanhada de atividade EMG tônica de amplitude crescente no tibial anterior do mesmo lado $(D)$ ou fenômeno de Strümpell positivo (calibração: 0,20mV, 1s). 
apresentavam distúrbios subietivos sensitivos; $13(50 \%)$ sinaís deficitarios motores moderados ou subietivos e $20(77 \%)$ sinais de liberaçăo piramidal diferentes do Fs, descobertos mediante a exame clírico ou de EMG (Tabela 1). Nenhum desses pacientes apresentava sinal de Babinski. Nove pacientes foram encaminhados ao laboratório de EMG com diagnósticos de moléstias que podiam estar direta ou indiretamente relaclonadas a sindrome piramidal (Tabela 2).

\section{COMENTARIOS}

Praticamente $4,5 \%$ dos pacientes encaminhados para realizar EMG no periodo de março a agosto-1987 apresentavam sinais de liberação piramidal diagnosticados mediante a pesquisa do FS. "O registro EMG dessa sincinesia de coordenação é de fácil realização e interpretação, não precisando de equipamentos especiais. Manifesta-se por EMG involuntário, com padrão de contração tônica, provocado pela extensão voluntária da coxa. Quando registrados com velocidades de varredura vagarosas, como $1 \mathrm{~s} /$ divisão, tomam aspecto de traçados de interferência, de duração aproximadamente igual ao movimento de extensão completa da coxa (Fig. 2D). O EMG do FS diferencia-se sem dificuldađe das contraçōes clônicas observadas nos tibiais anteriores, em $10 \%$ do grupo de pessoas normais e no lado normal de alguns casos do grupo de doentes. Todavia, $18(90 \%)$ dos casos do grupo de controle não apresentavam qualquer tipo de atividade EMG no tibial anterior durante a execução da manobra de Strümpell. Por conseguinte, o registro EMG do FS parece prova de valor para o diagnóstico de síndromes piramidais. Isto contrasta com a interpretação clínica desse sinal que, nos casos de lesões da via piramidal moderadas ou leves, frequentemente pode deixar dúvidas, agravadas quando faltam outros sinais de liberação piramidal, como o sinal de Babinski. Isto acontecia nos 26 casos estudados, nos quais a constatação do FS sob controle da EMG foi importante para comprovar objetivamente a presença dessa sincinesia de coordenação.

Excetuando-se os casos de esclerose lateral amiotrófica (ELA) e de esclerose múltipla (EM), os pacientes foram enviados ao laboratório de EMG com suspeita de apresentarem sindromes polineuríticas ou radiculares, provavelmente em consequêência dos sintomas sensitivos subjetivos que afetavam $18(69 \%)$ casos desse grupo. Essa hipótese diagnóstica pode ser favorecida pela constatação freqüente de reduçōes da neurocondução sensitiva em pacientes de idade avançada e em diabéticos, circunstâncias presentes no grupo de doentes estudados. A presença do FS, nesses casos, serviu para levantar a suspeita de que, pelo menos uma parte dessas queixas sensitivas, poderia ter origem central.

A avaliação pela EMG de déficits motores de etiologia piramidal oferece dificuldades, Daube 6 comenta a este respeito que «a ativação deficiente de neurônios motores nesses casos, traduzida por redução do número de potenciais de ação de unidades motoras que deflagram com vagar, é achado inespecifico que não pode ser utilizado para fins diagnósticos, visto que pode depender da colaboração precária, dor, ou outros disturbios do controle motor». Todavia, essas dificuldades podem ser superadas comparando-se os EMG conseguidos mediante o máximo esforço voluntário com os obtidos mediante a provocação de contraçōes reflexas, como o FS no tibial anterior, pois em casos de paresias piramidais esses EMG são quantitativamente iguais ou maiores, quando de origem reflexa (Fig. 1). Esta observação está de acordo com Grimby e Mannerz 11 que, estudando contrações leves voluntárias no homem, observaram que, em pacientes com lesões parciais da medula, o recrutamento ¿́ o mesmo, tanto quando depende de ativação reflexa como de ativação voluntária. Os sinais de liberação piramidal eram os mais freqüentes. $O$ registro $E M G$ das trepidaçōes como do clono, por serem facilmente avaliados clinicamente, tem menos interesse sob o ponto de vista diagnóstico. O registro de reflexos exaltados, que era o mais freqüente sintoma depois do $\mathrm{FS}$, é de difícil avaliação $\mathrm{EMG}$, como tem acontecido com as tentativas de avaliação do reflexo $\mathrm{H}$ para o diagnóstico de acometimento piramidal $8,13,15$ e extrapiramidal 3. O registro EMG das sincinesias tem utilidade, por serem movimentos anormais de aparecimento precoce e com significado diagnóstico preciso. O estudo EMG da marcha espástica, constatada clinicamente em 4 casos, apresenta dificuldades técnicas à EMG convencional. Freund 10 registra alteraçōes grosseiras e precoces durante a marcha em enfermos com acometimento do SNC, mediante técnicas de EMG caracterizadas pela co-ativação de músculos agonistas e antagonistas, em conseqüência do desaparecimento da inibição destes últimos durante marcha. O mesmo constatam Delwaide 7 e Dimitrijevic 9 durante movimentos voluntários alternados de flexão e extensão do pé. A espasticidade, registrada em forma de atividade espontânea contínua de potenciais de ação de unidades 
motoras nos músculos tibiais anteriores em três casos, é útil para levantar a suspeita de acometimento piramidal. Nesses casos, o acometimento é confirmado pela constatação do FS. O sinal da tríplice flexão, presente em três casos, é facilmente registrado à EMG e de fácil observasão clínica mas, quando isolado ou moderado, é difícil de avaliar, pois resulta de reflexo de defesa presente em pessoas normais.

Em relação ao diagnóstico clínico de alguns dos pacientes, o registro do FS serviu no caso com EM para confirmar esse diagnóstico. Em uma paciente com antecedentes de síndrome de Guillain-Barré, com persistência de dificuldades motoras nos membros inferiores, a constatação do FS, junto ao fato de não serem observados EMG de padrão neurogênico, serviu para sugerir que apresentava provável seqüela de mielite. No caso de ELA, a presença dessa sincinesia, junto ao registro de fasciculaçōes e sinais de degeneração axonal de unidades motoras, serviu para confirmar o diagnóstico e afastar polineuropatia periférica axonal. Foi importante a constatação do FS em uma paciente com antecedentes de mastectomia por câncer de mama e afetada de lombalgia, por suspeitar-se de possível metástase afetando a via piramidal. Nos 5 pacientes com diabetes mellitus, se o exame de EMG fosse orientado apenas à exploração de nervos periféricos, poderiam suas queixas sensitivas e/ou motoras serem relacionadas exclusivamente a multineuropatias periféricas. Em aproximadamente $4,5 \%$ dos enfermos enviados com hipóteses diagnósticas de moléstias do sistema nervoso periférico para fazer EMG, durante periodo de aproximadamente 5 meses, foi constatado o FS ao EMG e, por conseguinte, que apresentavam síndromes piramidais.

Esses resultados sugerem a inclusão da pesquisa, sob controle EMG, dessa sincinesia de coordenação no exame convencional dos músculos tibiais anteriores. A pesquisa do FS deve ser especialmente recomendada naqueles casos em que a EMG convencional $\mathrm{e}$ as medidas de condução nervosa não expliquem satisfatoriamente o quadro clínico dos pacientes, bem como deve ser obrigatória nos casos de esclerose lateral amiotrófica e em sindromes mielorradiculares e medulares.

\section{REFFERANCIAS}

1. Alonso JL - Fundamentos para interpretacão da eletromiografia clínica. Rev Méd IAMSPE (Sån Paulo) 17:39, 1986.

2. Alonso JL - Registro EMG do sinal de Strümpell (abstr.). Resumos X Congresso Brasíleiro de EEG e Neurofisiologia Clínica: Reunióes de Neurofísiologia Clínica 2:57. São Paulo, 1987.

3. Artieda J, Quesada P, Obeso JA, Martinez-Lage JM - Fisiopatologia de la rigidez en la entermedad de Parkinson: estudios eletrofisiológicos. In Obeso JA, Martínez-Lage (eds): Enfermedad de Farkinson y Movimientos Anormales. EUNSA, Pamplona, 1986, pg 49.

4. Barraquer-Bordás L - Neurologia Fundamental. Ed 3. Toray, Barcelona, 1976, pg 176.

5. Castaigne $\mathbf{P}-$ Clinical features of the pyramidal syndrome. In Desmedt JE (ed): New Developments in EMG and Clinical Neurophysiology, Vol. 3. Karger, Basel, 1973, pg 11.

6. Daube JR - Estudos eletrofisiológicos no diagnóstico e prognóstico das doenças do neurônio motor. In Aminoff MJ (ed): Eletrodiagnóstico. Interlivros, Rio de Janeiro, 1987, pg 1.

7. Delwalde PJ - Contribution of human reflex studies to the understanding and management of the pyramidal syndrome. In Shahani BT (ed): Electromyography in CNS Disorders: Central EMG. Butteworth, Boston, 1984, pg 77.

8. Lesmedt JE - New Developments in EMG and Clinical Neurophysiology, Vol 3. Karger, Basel, 1973.

9. Dimltrijevic MR - Chronic upper motor neuron syndromes. In Desmedt JE (ed): New Developments in EMG and Chinical Neurophysiology, Vol. 3. Karger, Basel, 1973, pg 111.

10. Freund HJ - The Dathophysiology of central paresis. In Struppler $\mathbf{A}$, Weindl A (eds): Electromyography and Eroked Patentials. Springer, Berlin, 1985, pg 19.

11. Grimby L, Mannerz $J$ - Tonic and phasic recruitment order of motor units in man under normal and pathological conditions. In Desmedt JE (ed): New Developments in EMG and Clinical Neurophysiology, Vol 3, Karger, Basel, 1973.

12. Obeso JA, Martinez-Lage JM - Enfermedad de Parkinson y Movimientos Anormales. LUNSA, Pamplona, 1986.

13. Shahani BT (ed) - Electromyography in CNS Disorders: Central EMG. Butterworth, Boston, 1984.

14. Strümpełl A - Exámen de las Enfermedades Nerviosas. Paracelso, Madrid, 1924, pg 27.

15. Struppler A, Weindl A - Electromyography and Evoked Potentials. Springer, Berlin, 1985. 